\title{
Lipopolysaccharides from Mesorhizobium huakuii and Mesorhizobium ciceri: chemical and immunological comparative data
}

\author{
Adam Choma
}

\section{Department of General Microbiology, Maria Curie-Sktodowska University, Lublin, Poland}

Received: 09 July, 2002; revised: 16 October, 2002; accepted: 10 December, 2002

Key words: Mesorhizobium huakuii, Mesorhizobium ciceri, lipopolysaccharides, immunobloting, fatty acids, sugars, 2,3-diamino-2,3-dideoxy-D-glucose

Lipopolysaccharides of two Mesorhizobium species of different host specificity were compared: $M$. huakuii and $M$. ciceri. $M$. huakuii sp. was represented by five strains with special consideration of $M$. huakuii IFO $15243^{\mathrm{T}}$.

SDS/PAGE profiles revealed that all M. huakuii LPS preparations contained low molecular mass fractions (LPS-II) of the same molecular size. All of lipopolysaccharides contained high molecular mass fractions (LPS-I). However, the high molecular mass fraction from each strain possessed an individual molecular size distribution pattern. The crossreactivity of blotted lipopolysaccharides with rabbit polyclonal antibodies against Mesorhizobium huakuii IFO $15243^{\mathrm{T}}$ whole bacteria indicated the presence of common epitope(s) within the investigated Mesorhizobium huakuii strains. Moreover, LPS from M. huakuii S52 also reacted with anti M. ciceri HAMBI 1750 serum showing that there are epitopes common for different mesorhizobial species.

LPS isolated from Mesorhizobium huakuii strain IFO $15243^{\mathrm{T}}$ contained neutral sugars: L-6-deoxytalose, L-rhamnose, D-galactose and D-glucose, aminosugars: D-quinovosamine, D-glucosamine, D-2,3-diamino-2,3-dideoxyglucose and D-galacturonic and D-glucuronic acids. In the LPS preparation, fatty acids typical for Mesorhizobium strains were detected. 3-Hydroxydodecanoic, 3-hydroxy-iso-tridecanoic, 3-hydroxyeicosanoic, 3-hydroxyheneicosanoic and 3-hydroxydocosenoic acids were the major amide linked fatty acids, while iso-heptadecanoic, eicosanoic, docosenoic, as well as 27-hydroxyoctacosanoic and 27-oxooctacosanoic acids were the dominant ester linked fatty residues.

\footnotetext{
${ }^{\otimes}$ Corresponding author: Adam Choma, Department of General Microbiology, Maria Curie-Skłodowska University, 19 Akademicka St., 20-033 Lublin, Poland; tel.: (48 81) 537 5981; fax: (48 81) 537 5959; e-mail: achoma@biotop.umcs.lublin.pl
}

Abbreviations: Cps, capsular polysaccharides; EPS, exopolysaccharides; Kdo, 3-deoxy-D-mannooctulosonic acid; LPS, lipopolysaccharides. 
Bacteria from the Mesorhizobium species are classified as other rhizobia in the $\alpha$ subgroup of Proteobacteria and are Gram-negative soil microorganisms that induce nitrogen fixing nodules on roots of leguminous plants (Jarvis et al., 1997; Malek \& Sajnaga 1999; Tighe et $a l ., 2000)$. Their surface components, especially polysaccharides, such as acidic exopolysaccharides (EPS), lipopolysaccharides (LPS), capsular polysaccharides (CPS), and cyclic $\beta$-glucans, play an important role during the free-living stage in soil environment and especially during the development of symbiosis. Changes of environmental growth conditions affect the cell surface polysaccharide constitution (Kannenberg et al., 1998; Kannenberg et al., 2001).

Mesorhizobium huakuii strains induce the formation of indeterminate nodules on host roots of Astragalus sinicus. An acidic EPS is required for the successful establishment of this type of nodules. Hisamatsu and coworkers (1997) have proposed that the backbone structure of $M$. huakuii-My6 EPS is very similar to that of EPS-13336 from Sinorhizobium meliloti. Also, the cyclic glucans secreted to the medium by M. huakuii My6 have a distribution pattern of ring size resembling that of S. meliloti.

In comparison with other rhizobial genera, Mesorhizobium LPSs have not been detailed. So far, the structure of two O-specific polysaccharides (Russa et al. 1995; Choma et al., 2000), cellular fatty acid composition and lipopolysaccharide fatty acid analyses, as well as characteristics of water and phenol soluble fractions of LPS from Mesorhizobium have been described (Yokota, et al., 1993; Russa et al., 1995a; Choma et al., 2000a).

Systematic studies of Mesorhizobium lipid A have not been completed. However, several authors have indicated that the sugar backbone of mesorhizobial lipid A represents the DAG-type (meaning, it is composed of 2,3-diamino-2,3-dideoxy-D-glucose disaccharide) (Russa et al., 1995a, Urbanik-Sypniewska et al., 2000). This type of lipid A has been found in Bradyrhizobium sp. (Lupinus) (Mayer et al., 1989) but not in any other Rhizobiaceae LPS preparations.

For a comparative analysis of LPSs we have selected two Mesorhizobium species of different host specificity. $M$. huakuii creates nitrogen fixing nodules on Astragalus sinicus and M. ciceri on Cicer arietinum. Special attention was focused on the chemical and immunochemical characteristics of lipopolysaccharide of Mesorhizobium huakuii IFO $15243^{\mathrm{T}}$ (type strain in the Culture Collection of the Institute of Fermentation, Osaka), as compared with other $M$. huakuii and $M$. ciceri lipopolysaccharides using appropriate rabbit antisera. The strains selected for the analysis were isolated in different regions of the world. Four of M. huakuii strains are from China, one is from Japan, whereas $M$. ciceri bacteria were isolated from Spanish soil. The diversified geographic origins of the strains give a good opportunity to conduct comparative studies of bacteria (strains) from the same species or belonging to the same genus yet originating from places very distant from one another.

\section{MATERIALS AND METHODS}

Bacterial strains and their sources are listed in Table 1. The bacteria were grown at $28^{\circ} \mathrm{C}$ in liquid mannitol-yeast extract medium 79CA (Vincent, 1970) and were aerated by vigorous shaking. LPSs were extracted from the cells by the hot phenol-water procedure, with the modification of Johnson \& Perry (1976).

For sugar analysis, LPS was hydrolysed with $2 \mathrm{M}$ TFA $\left(100^{\circ} \mathrm{C}, 4 \mathrm{~h}\right)$. The sugars were converted into alditol acetates. Acidic sugars were liberated by methanolysis, then carboxyl reduced with $\mathrm{NaBD}_{4}$, hydrolysed with $2 \mathrm{M}$ TFA $\left(100^{\circ} \mathrm{C}, 4 \mathrm{~h}\right)$, repeatedly reduced with $\mathrm{NaBD}_{4}$, finally peracetylated and analysed as alditol acetates (Russa et al., 1995a). For the analysis of amino sugars in the form of peracetylated amino alditols, LPS sample was hydrolysed with $4 \mathrm{M} \mathrm{HCl}$ for $8 \mathrm{~h}$ at $100^{\circ} \mathrm{C}$ and 
Table 1. Bacterial strains used in this study

\begin{tabular}{|c|c|c|c|}
\hline $\begin{array}{l}\text { Mesorhizobium } \\
\text { strain }\end{array}$ & $\begin{array}{l}\text { Other possible designa- } \\
\text { tions of the strain }\end{array}$ & $\begin{array}{l}\text { Host plant and geographic } \\
\text { origin }\end{array}$ & Source and references \\
\hline huakuii IFO $15243^{\mathrm{T}}$ & CCBAU 2603 & Astragalus sinicus, China & $\begin{array}{l}\text { IFO } \\
\text { Nuswantara et al., } 1997\end{array}$ \\
\hline huakuii IFO 15244 & My-3 & $\begin{array}{l}\text { Astragalus sinicus subsp. } \\
\text { rengei, Japan }\end{array}$ & $\begin{array}{l}\text { IFO } \\
\text { Yokota et al., } 1993\end{array}$ \\
\hline huakuii 38 & & Astragalus sinicus, China & $\begin{array}{l}\text { CCBAU } \\
\text { Chen et al., } 1991\end{array}$ \\
\hline huakuii S52 & & Astragalus sinicus, China & $\begin{array}{l}\text { CCBAU } \\
\text { Chen et al., } 1991\end{array}$ \\
\hline huakuii Pl-52 & & Astragalus sinicus, China & $\begin{array}{l}\text { CCBAU } \\
\text { Chen et al., } 1991\end{array}$ \\
\hline $\begin{array}{l}\text { ciceri HAMBI } 1750 \\
\text { Type strain }\end{array}$ & $\begin{array}{l}\text { UPM-Ca7 } \\
\text { ATCC } 51585^{\mathrm{T}}\end{array}$ & Cicer arietinum, Spain & $\begin{array}{l}\text { HAMBI } \\
\text { Nour et al., } 1994\end{array}$ \\
\hline
\end{tabular}

CCBAU, Culture Collection of Beijing Agricultural University, Beijing, People's Republic of China; HAMBI, Culture Collection of the Department of Microbiology, University of Helsinki, Finland; IFO, Culture Collection of the Institute of Fermentation Osaka, Japan.

then $\mathrm{N}$-acetylated prior to the reduction. The absolute configurations of sugars were determined using (-)-2-butanol for glycoside preparation (Gerwig et al., 1978).

Fatty acids were released and converted into methyl esters by methanolysis $(2 \mathrm{M} \mathrm{HCl}$ in methanol, $85^{\circ} \mathrm{C}, 16 \mathrm{~h}$ ) of freeze-dried LPS. Preparations of fatty acid methyl esters were trimethylsilylated with SIL-MIX (POCh, Poland) and the reaction mixture was directly injected into a GC-MS apparatus. Ester- and amide-linked fatty acids were distinguished using a method described by Wollenweber and Rietschel (1990) or according to Sonesson et al. (1994). Authentic 4-oxo and 27-oxo fatty acids for comparative chromatographic analysis were isolated from appropriate LPS preparations (M. loti NZP2213 (Russa et al., 1995a) and L. pneumophila serogroup 1 LPS, respectively).

Alditol acetates and fatty acid methyl esters were analysed routinely with a HewlettPackard gas chromatograph (model HP 5890A) equipped with a capillary column (HP-5MS, $30 \mathrm{~m} \times 0.25 \mathrm{~mm}$ ) and connected to a mass selective detector (MSD model HP 5971). The carrier gas was helium and the temperature program was initially $150^{\circ} \mathrm{C}$ for 5 min, then raised to $310^{\circ} \mathrm{C}$ at a ramp rate of $5^{\circ} \mathrm{C} / \mathrm{min}$, final time $20 \mathrm{~min}$.

Polyacrylamide gel electrophoresis (PAGE) was performed with sodium dodecyl sulphate (Laemmli, 1970) and the slab was silver stained after oxidation with periodic acid (Tsai \& Frasch, 1982).

Two polyclonal rabbit antisera were obtained against whole $M$. huakuii IFO $15243^{\mathrm{T}}$ cells and $M$. ciceri HAMBI 1750 . Rabbits were injected with $0.2 \mathrm{ml}$ of temperature killed bacteria (about $10^{9}$ bacteria per $\mathrm{ml}$ ). The bacteria were washed twice with and resuspended in phosphate-saline buffer, $\mathrm{pH}$ 7.0, prior to boiling. The rabbits were injected weekly, thrice. Three days after the last injection, the rabbits were bled and antisera were prepared according to Carlson and coworkers (1987). Small portions of the ready to use antisera were stored at $-20^{\circ} \mathrm{C}$.

Lipopolysaccharides separated by SDS/ PAGE were transferred electrophoretically from the gel slab to Immobilon P (Millipore). After reaction with the appropriate antiserum the electroblotted material was immunostained with goat anti-rabbit antibodies coupled to alkaline phosphatase (Sigma). The blots were developed with nitroblue tetra- 
zolium and 5-bromo-4-chloro-3-indolylphosphate toluidine (Sigma).

\section{RESULTS AND DISCUSSION}

The yield of the water soluble lipopolysaccharide from $M$. huakuii IFO $15243^{\mathrm{T}}$ strain was approximately $0.3 \%$ of the bacterial dry mass. A small portion of LPS was found in the phenol phase. Because of insufficient quantity, the material was not analysed further. The neutral, amino and acidic sugar components of $M$. huakuii IFO $15243^{\mathrm{T}}$ LPS are given in Table 2. The LPS is mainly composed of
(DAG) and in comparison with the known rhizobial lipopolysaccharide structures. Possibly it is due to strong hydrolysis conditions used for liberation of these saccharides. The conditions used in our experiment were directed to neutral sugar hydrolysis and were unappropriate for liberation of Kdo.

In our previous studies it was shown that deoxysugars were the only components of the O-specific chain of $M$. huakuii IFO $15243^{\mathrm{T}}$ LPS (Choma et al., 2000). The molar ratio of L-6-deoxytalose to L-rhamnose in the O-specific polysaccharide was 2:1. The same ratio, estimated for intact LPS, was nearly 1:1. This divergence can be explained when

Table 2. Neutral, acidic and aminosugar composition of LPS from Mesorhizobium huakuii IFO $15243^{\text {T }}$

\begin{tabular}{lrll}
\hline Component & $\begin{array}{c}\text { Amount } \\
\mu \mathrm{g} / \mathrm{mg} \text { LPS }\end{array}$ & Component & $\begin{array}{c}\text { Amount } \\
\mu \mathrm{g} / \mathrm{mg} \text { LPS }\end{array}$ \\
\hline Neutral sugars & & Acidic sugars & \\
L-6-Deoxytalose & 92.6 & D-Galactouronic acid & 22.7 \\
L-Rhamnose & 110.1 & D-Glucuronic acid & 13.6 \\
D-Mannose & 6.8 & & \\
D-Glucose & 127.2 & Amino sugars & \\
D-Galactose & 155.1 & & 12.7 \\
L-Glycero-D-mannoheptose & 59.0 & D-Quinovosamine & 10.6 \\
Kdo & 2.3 & D-Glucosamine & 46.2 \\
\end{tabular}

6-deoxytalose and rhamnose, both sugars in L-configuration. D-Galactose and D-glucose, as well as L-glycero-D-manno-heptose, were also found in significant amounts. The absolute configuration of the last mentioned sugar was estimated by chromatographic comparison with the sugars released from Azospirillum lipoferum SpBr17 LPS. This microorganism contains both L-glycero- and D-glycero-D-mannoheptose within its LPS (Choma et al., 1987). The amount of 3-deoxy-D-manno-octulosonic acid (Kdo), estimated by the GLC method as appropriate alditol acetate, seems to be too low in relation to the amount of aminosugars, especially 2,3-diamino-2,3-dideoxyglucose one takes into consideration that L-rhamnose is also a constituent of the core oligosaccharide. The heptose, the hexoses, D-glucuronic acid and aminosugars, with the exception of DAG, are presumably components of the core oligosaccharide as well. Preliminary studies of lipid A from the IFO $15243^{\mathrm{T}}$ strain isolated by mild acid hydrolysis (1\% acetic acid, $1 \mathrm{~h}, 100^{\circ} \mathrm{C}$ ) showed that DAG is the only aminosugar present in that fraction of LPS. Similar data have been published by Russa et al. (1995a) and Urbanik-Sypniewska et al. (2000) for LPS from M. loti HAMBI 1129 and HAMBI 1148, respectively. 
SDS/PAGE analysis of the LPSs from $M$. huakuii IFO $15243^{\mathrm{T}}$, IFO 15244 , S52, Pl-52 and 38 strains, as well as $M$. ciceri HAMBI 1750 , indicated a high heterogeneity of these preparations (Fig. 1). All lipopolysaccharides

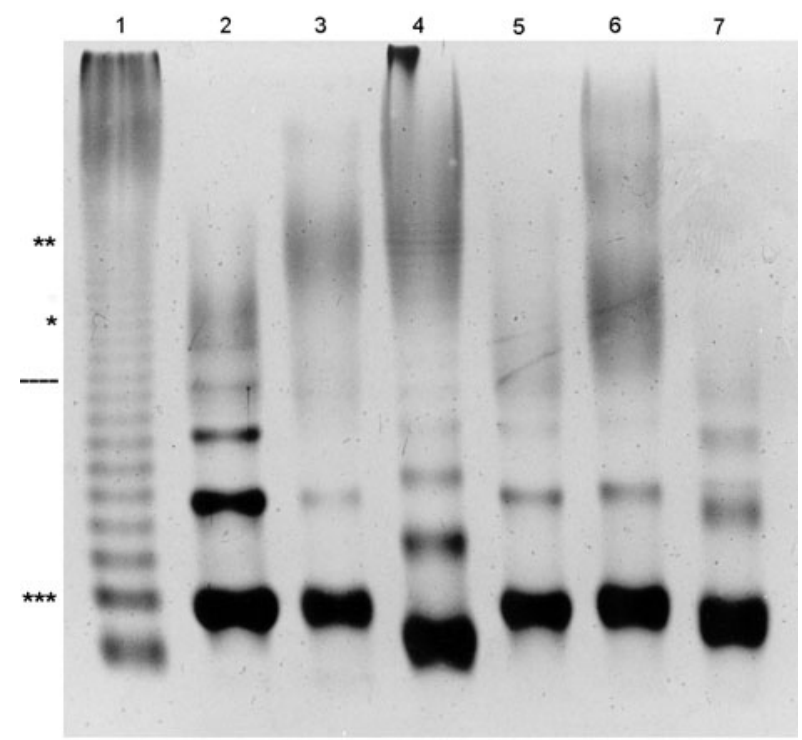

Figure 1. Silver-stained SDS/PAGE profiles of LPS.

Lane 1, Salmonella montevideo SH94. (standard); lane 2, M huakuii IFO $15243^{\mathrm{T}}$; lane $3, M$. huakuii IFO 15244 ; lane 4, M. ciceri HAMBI 1750; lane 5, M. huakuii 38; lane 6, M. huakuii S52; lane 7, M. huakuii Pl-52. The positions of LPS-I and LPS-II were separated by a dashed line. (-). Asterisk. $\left({ }^{*}\right)$ indicates the position of the most abundant HMW LPS-I fractions of $M$. huakuii IFO $15243^{\mathrm{T}}$. Double asterisks. $\left({ }^{* *}\right)$ indicate the position of the most abundant HMW LPS-I fractions of M. huakuii IFO 15244. Triple asterisks indicate the position of the simplest incomplete LPS (basic core and lipid A).

contained high molecular mass fractions (LPS-I). The distribution of molecular weight of LPS-I was different for each preparation (Fig. 1). For example, the most intense bands of M. huakuii IFO $15243^{\mathrm{T}}$ LPS-I had the same mobility as band No. 15 (counting from the bottom of the slab, indicated in Fig. 1 with an asterisk) from $S$. montevideo SH 94 LPS. The average mass of LPS-I molecules from M. huakuii IFO 15244 was higher than that from $M$. huakuii IFO $15243^{\mathrm{T}}$ LPS and these molecules migrated in a similar manner to the molecules from band No. 20 of $S$. montevideo SH 94 LPS (indicated with two asterisks in Fig. 1). The smallest-sized molecules within LPS-I fractions were found in LPS from M. huakuii Pl-52. Intensely stained bands are present in the bottom of the SDS/PAGE electrophoregrams. Low molecular mass fractions in all $M$. huakuii preparations migrated parallel to the band No. 2 of S. montevideo LPS (three asterisks). $M$. huakuii LPS preparations, except for IFO 15244 and S52, showed intensely stained second and third low molecular mass bands. The low molecular mass LPSs from $M$. ciceri HAMBI 1750 were found to be even smaller than those from $M$. huakuii, the electrophoregram of this slot is shifted a little down.

It is interesting to note that densitometric analysis of the gel (not shown) showed that the lowest molecular size LPS fractions were also the most abundant and represented more than $30 \%$ of LPS in each case.

Figure 2 shows immunoblot of the gel probed with a polyclonal rabbit antiserum against M. huakuii IFO $15243^{\mathrm{T}}$. LPSs of all

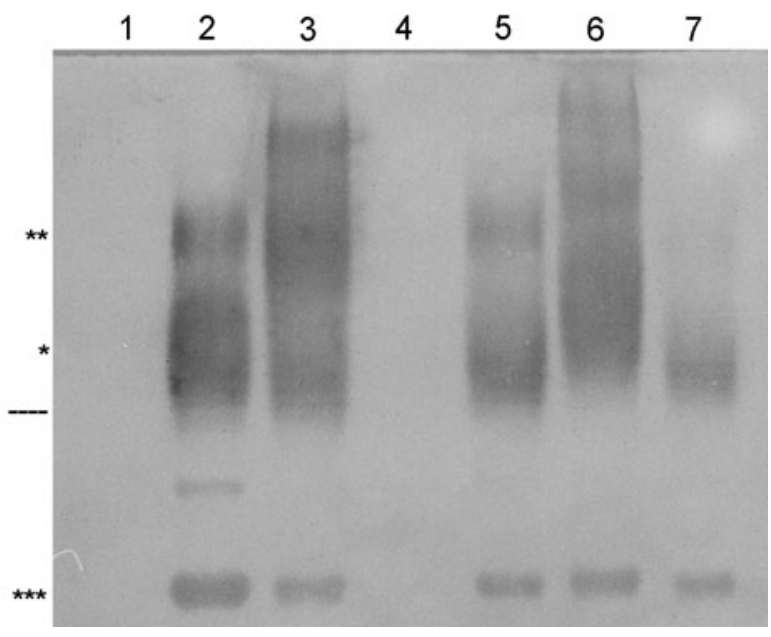

Figure 2. Immunoblot probed with polyclonal rabbit antiserum against M. huakuii IFO $15243^{\mathrm{T}}$ temperature killed whole bacteria.

Lane 1, Salmonella montevideo SH94 (standard); lane 2, M. huakuii IFO $15243^{\mathrm{T}}$; lane 3, M. huakuii IFO 15244; lane 4, $M$. ciceri HAMBI 1750; lane 5, M. huakuii 38; lane 6, M. huakuii S52; lane 7, M. huakuii Pl-52. Symbols along the left side of the blot are defined in the legend to Fig. 1. 
M. huakuii species could be detected with this antiserum. No other LPS preparation (LPS M. ciceri HAMBI 1750 and S. montevideo SH 94) was immunostained. Rabbit antisera react strongly with the epitopes from O-polysaccharides and weakly with other LPS fractions. This feature is well known and has been described for Rhizobium and Bradyrhizobium. The majority of antibodies are directed against the O-chain epitopes and react slightly with LPS composed only of lipid A and the core (Kannenberg et al., 1998). In the case of Mesorhizobium, incomplete lipopolysaccharides, containing the core oligosaccharide and lipid A, were stained very weakly while some bands remained unlabeled (Fig. 2). This immunostaining enabled us to visualise the molecular mass distribution of $M$. huakuii high molecular fraction LPSs even better than silver staining. Moreover, because the immunoreaction with the so called "rough" LPS is slight, one can conclude that the LPS material which migrated faster than $S$. montevideo $\mathrm{SH}$ 94 LPS band No. 10 (counting from the bottom of the slab, marked with a dashed line) belonged to the set of incomplete $M$. huakuii LPS molecules (LPS-II). If that indeed were the case, then the Mesorhizobium LPS bands visible in the bottom part of the slab are representative of fine differences in the core structure. The oligosaccharides could have originated at different stages of the core biosynthesis pathway. The LPS probes of M. ciceri HAMBI 1750 and M. huakuii S52 reacted with a rabbit antiserum obtained against $M$. ciceri HAMBI 1750 whole bacteria. Also, this antiserum reacted positively exclusively with LPS-I fractions (Fig. 3). It is worth noting that $M$. huakuii Pl-52 as the only one among $M$. huakuii strains crossreacted with antiserum against $M$. ciceri although its reaction with antiIFO $15243 \mathrm{~T}$ was the same as with other strains.

A polyphasic approach to the determination of the taxonomic position of mesorhizobia evidently indicates a very high similarity between M. loti and M. ciceri (Wang et al., 1999).
Strains of $M$. huakuii were found to be closely related to both of them (Jarvis et al., 1997; Malek \& Sajnaga 1999; Wang et al., 1999). Although $M$. huakuii and M. ciceri strains are almost identical from the genetic and chemotaxonomic (Choma et al., 2000a; Tighe et al., 2000) point of view, they effectively create nitrogen fixing nodules in differing host plants (Astragalus sinicus and Cicer arietinum, respectively). The studied strains were isolated in different regions of the world, i.e. M. huakuii strains are from China and Japan, whereas M. ciceri HAMBI 1750 was isolated from Spanish soil (Chen et al., 1991; Yokota et $a l .$, 1993; Nour et al., 1994). Neither the geographic distance separating their places of origin nor the different host plants were a barrier for the development of the same epitopes

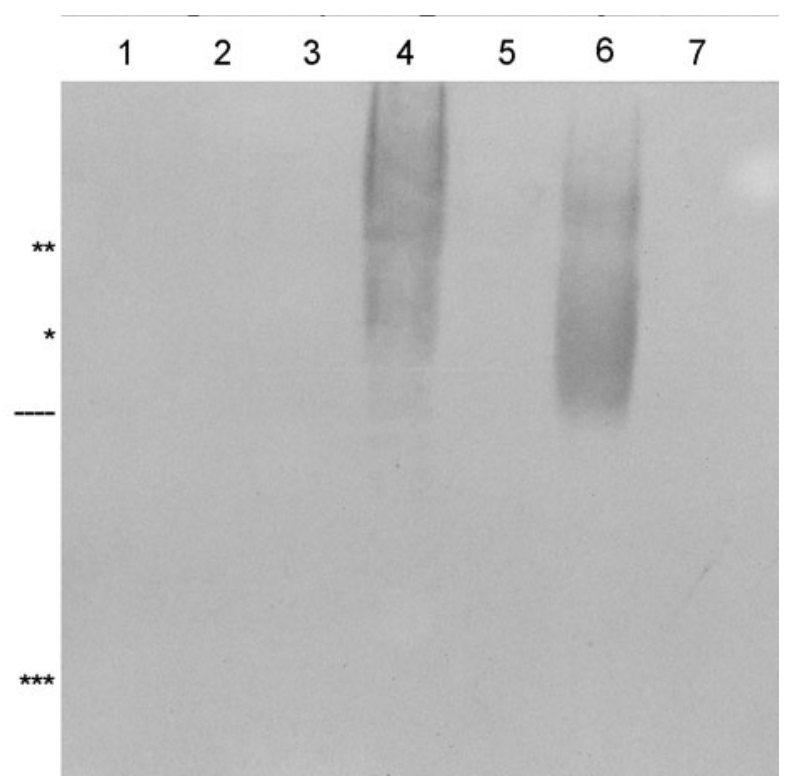

Figure 3. Immunoblot probed with polyclonal rabbit antiserum against M. ciceri HAMBI 1750 temperature killed whole bacteria.

Lane 1, Salmonella montevideo SH94. (standard); lane 2, M. huakuii IFO $15243^{\mathrm{T}}$; lane 3, M. huakuii IFO 15244; lane 4, M. ciceri HAMBI 1750; lane 5, M. huakuii 38; lane 6, M. huakuii S52; lane 7, M. huakuii PL-52. Symbols are defined in the legend to Fig. 1.

during the process of lipopolysaccharide biosynthesis in the HAMBI 1750 and Pl-52 strains. Thus, the results of immune reactions between the described LPS preparations and 
M. ciceri HAMBI 1750-specific antibodies may be explained by similarities among the genes determining O-chain synthesis.

The crossreactivity of the antiserum against the IFO 15243 T strain with all $M$. huakuii LPS preparations tested seems to be self-evident taking into consideration their close taxonomic positions (Chen et al., 1991). Reactions with the anti IFO $15243 \mathrm{~T}$ serum indicate a similarity among the structures of the investigated lipopolysaccharides. A preliminary chemical analysis (not presented) pointed to main components shared by the lipopolysaccharides tested (6-deoxytalose, rhamnose, glucose and galactose). A similar chemical structure of LPS fragments renders the search of biochemical pathways leading to the synthesis of whole LPS molecules much more feasible.
The rhizobial O-chain structures published earlier revealed a high degree of diversity. The latest studies on modification of Rhizobium LPS during bacteroid development suggest that instead of a specific interaction or a specific site within the O-chain structure, the hydrophobic character of the entire LPS molecule is the crucial factor for normal development of symbiosis (Kannenberg \& Carlson, 2001). The bacteria need the complete lipopolysaccharides (i.e. composed of lipid A, core and O-specific chain) to effectively infect the host plants. Therefore, any mutation in the genes responsible for core synthesis leads to the creation of O-chain deprived nod mutants (Campbell et al., 2002). Moreover, neither the appropriate structure nor the appropriate length of the O-chain but solely its presence in the LPS in a hydrophobic form is es-

Table 3. Fatty acid composition of LPS from Mesorhizobium huakuii IFO $15243^{\text {T }}$

\begin{tabular}{lclc}
\hline Component & Amount & Component & Amount \\
Amide linked fatty acids & $\mu \mathrm{g} / \mathrm{mg}$ LPS & Ester linked fatty acids & $\mu \mathrm{g} g$ LPS \\
\hline 3-OH-12:0 & 40.0 & $12: 0$ & $\operatorname{Tr}$ \\
3-OH-i-13:0 & 69.0 & $14: 0$ & $\operatorname{Tr}$ \\
3-OH-14:0 & 1.6 & $16: 0$ & 4.3 \\
3-OH-16:0 & 11.2 & $i-17: 0$ & 8.1 \\
3-OH-18:0 & 2.9 & $18: 1$ & 0.2 \\
3-OH-19:0 & Tr & $18: 0$ & 0.4 \\
3-OH-20:0 & 64.4 & $19: 1$ & 0.7 \\
3-OH-i-21:0 & 8.8 & $20: 0$ & 7.8 \\
3-OH-22:1 & 3.4 & $20: 1$ & 1.9 \\
3-OH-22:0 & 9.1 & $21: 0$ & 0.8 \\
3-OH-23:1 & 1.4 & $22: 1$ & 6.8 \\
& & $22: 0$ & 1.8 \\
4-oxo-20:0 & 5.8 & $23: 1$ & 1.1 \\
4-oxo-i-21:0 & 1.4 & $28: 1$ & 3.2 \\
4-oxo-22:0 & 1.2 & & 71.9 \\
& & $27-\mathrm{OH}-28: 0$ & 1.4 \\
& & $29-\mathrm{OH}-30: 0$ & 5.4 \\
& & &
\end{tabular}


sential in the process of nodulation (Kannenberg et al., 1998; Räsänen et al., 1997). Indeed, in the case of the discussed $M$. huakuii lipopolysaccharides, each preparation possesses an individual pattern of length distribution of the O-chain.

The fatty acid composition of LPS from $M$. huakuii IFO $15243^{\mathrm{T}}$ is shown in Table 3 . All 3-hydroxy fatty acids were found to be amide linked. 3-Hydroxydodecanoic, 3-hydroxy-isotridecanoic, 3-hydroxyeicosanoic and 3-hydroxydocosenoic acids were the major fatty acids. Additionally, 3-hydroxyheneicosanoic and 3-hydroxydocosenoic acids were present in significant amounts. Similarly to the $M$. ciceri and other Mesorhizobium strains (Choma et al., 2000a), M. huakuii IFO $15243^{\mathrm{T}}$ lipopolysaccharide contained amide bound 4-oxo fatty acids (Table 3). However, only 4-oxo-20:0 was present in quantities comparable to the amount of 3-hydroxy fatty acids. Others were present in trace amounts. Moreover, a few nonpolar ester linked fatty acids were identified but only three of them, namely: $i-17: 0$, 20:0 and 22:1, occurred in significant amounts. The total amount of nonpolar fatty acids is less than half the quantity of the main ester bound 27-OH-octacosanoic acid. This fatty acid was accompanied by 29-OH-30:0 and 27-oxo-28:0 acids. The presence of $\omega-1$ hydroxy fatty acids with hydrocarbon chains almost twice as long as "the usual" (i.e. 16:0, 18:0) fatty acids is typical for Rhizobium and Bradyrhizobium with the only exception of Azorhizobium caulinodans (Bath et al., 1991). Furthermore, the presence of 27-oxo-28:0 acid is characteristic for Mesorhizobium species (Choma et al., 2000a). Assuming that lipid A from $M$. huakuii LPS is composed of 2,3-diamino-2,3-dideoxyglucose exclusively, one might conclude that the mentioned ester linked fatty acids must create acyloxyacyl residues. Such acyloxyacyl moieties have, in fact, been described for Rhizobium etli CE3 lipid A (Que et al., 2000; 2000a). Previously, it was believed that bacteria from the Rhizobiaceae family can not synthesise acyloxyacyl resi- dues (Bhat et al., 1994). The above mentioned hypothesis should be verified experimentally. Therefore, further studies of the M. huakuii lipid A are in progress.

The author is grateful to Dr. J. Kutkowska for help with immunoblot experiments and to Dr. T. Urbanik-Sypniewska for critical reading of the manuscript and helpful discussion.

\section{R E F E R E N C ES}

Bhat, RU, Forsberg LS, Carlson RW. (1994) Structure of lipid A component of Rhizobium leguminosarum bv phaseoli lipopolysaccharide. J Biol Chem.; 269: 14402-10.

Bhat UR, Mayer H, Yokota A, Hollingsworth RI, Carlson RW. (1991) Occurrence of lipid A variants with 27-hydroxyoctacosanoic acid in lipopolysaccharides from members of the family Rhizobiaceae. J Bacteriol.; 173: 2155-9.

Campbell GRO, Reuhs BL, Walker GC. (2002) Chronic intracellular infection of alfalfa nodules by Sinorhizobium meliloti requires correct lipopolysaccharide core. Proc Natl Acad Sci U S A.; 99: 3938-43.

Carlson RW, Kalembasa S, Turowski D, Pachori P, Noel KD. (1987) Characterization of the lipopolysaccharide from a mutant of Rhizobium phaseoli which is defective in infection thread development. J Bacteriol.; 169: 4923-8.

Chen WX, Li GS, Qi YL, Wang ET, Li JL. (1991) Rhizobium huakuii sp. nov. isolated from the root nodules of Astragalus sinicus. Int $J$ Syst Bacteriol.; 41: 275-80.

Choma A, Russa R, Mayer H, Lorkiewicz Z. (1987) Chemical analysis of Azospirillum lipopolysaccharides. Arch Microbiol.; 146: $341-5$.

Choma A, Sowiński P, Mayer H. (2000) Structute of the O-specific polysaccharide of Mesorhizobium huakuii IFO 15243T. Carbohyd Res.; 329: 459-64. 
Choma A, Urbanik-Sypniewska T, Russa R, Kutkowska J, Mayer H. (2000a) Occurrence and taxonomic significance of oxo-fatty acids in lipopolysaccharides from members of Mesorhizobium. System Appl Microbiol.; 23: 185-90.

Gerwig GJ, Kamerling JP, Vliegenthart JFG. (1978) Determination of the D and L configuration of neutral monosaccharides by high-resolution capillary GLC. Carbohydr Res.; 62: 349-57.

Hisamatsu M, Nomura S, Shutsrirung A, Obata H, Teranishi K, Yamada T, Nusawantara S, Yamashita M, Murooka Y. (1997) Structural charactrization of a new acidic exopolysaccharide and cyclic $(1 \rightarrow 2) \beta$-glucan produced by Rhizobium huakuii forming nodules on Astragalus sinicus. J Ferment Bioengin.; 83: 315-20.

Jarvis BDW, Van Berkum P, Chen WX, Nour SM, Fernandez MP, Cleyet-Marel JC, Gillis M. (1997) Transfer of Rhizobium loti, Rhizobium huakuii, Rhizobium ciceri, Rhizobium mediteraneum, Rhizobium tianshanense to Mesorhizobium gen nov. Int $J$ Syst Bacteriol.; 47: 895-8.

Johnson KG, Perry MB. (1976) Improved techniques for the preparation of bacterial lipopolysaccharides. Can J Microbiol.; 22: 29-34.

Kannenberg EL, Carlson RW. (2001) Lipid A and O-chain modifications cause Rhizobium lipopolysaccharides to become hydrophobic during bacteroid development. Mol Micribiol.; 39: 379-91.

Kannenberg EL, Reuhs BL, Forsberg S, Carlson RW. (1998) Lipopolysaccharides and K-antigens: their structure biosynthesis and functions. In Rhizobiaceae. Spaink HP, Kondorosi A, Hooykaas PJJ, eds, pp 81-96. Kluwer Acad Publ, Dordrecht, Boston, London.

Laemmli UK. (1970) Cleavage of structural proteins during the assembly of the head of bacteriophage T4. Nature.; 227: 680-5.

Malek W, Sajnaga E. (1999) Current taxonomy of the Rhizobiaceae. Acta Microbiol Polon.; 2: 109-11.
Mayer H, Krauss JH, Urbanik-Sypniewska T, Puvanesarajah V, Stacey G, Auling G. (1989) Lipid A with 2,3-diamnio-2,3-dideoxy-glucose in lipopolysaccharides from slow-growing members of Rhizobiaceae and from "Pseudomonas carboxydovorans". Arch Microbiol.; 151: $111-6$.

Nour SM, Fernandez MP, Normand P, CleyetMarel J-C. (1994) Rhizobium ciceri sp. no.v consisting of strains that nodulate chickpeas. (Cicer arietinum L). Int J Syst Bacteriol.; 44: 511-22.

Nuswantara S, Fujie M, Sukiman HI, Yamashita M, Yamada T, Murooka Y. (1997) Phylogeny of bacterial symbionts of the leguminous tree Acacia mangium. J Ferment Bioeng.; 84: 511-8.

Que NLS, Lin S, Cotter RJ, Raetz CRH. (2000) Purification and mass spectrometry of six lipid A species from the bacterial endosymbiont Rhizobium etli. J Biol Chem.; 275: 28006-16.

Que NLS, Ribeiro AA, Raetz CRH. (2000a) Two-dimentional NMR spectroscopy and structures of six lipid A species from Rhizobium etli CE3. J Biol Chem.; 275: 28017-27.

Räsänen LA, Russa R, Urbanik T, Choma A, Mayer H, Lindstrm K. (1997) Characterization of two lipopolysaccharide type isolated from Rhizobium galegae. Acta Biochim Polon.; 44: 819-26.

Russa R, Urbanik-Sypniewska T, Shashkov AS, Kochanowski H, Mayer H. (1995) The structure of the homopolymeric O-specific chain from the phenol soluble LPS of the Rhizobium loti type strain NZP2213. Carbohydr Polymers.; 27: 299-303.

Russa R, Urbanik-Sypniewska T, Lindstrm K, Mayer H. (1995a) Chemical characterization of two lipopolysaccharide species isolated from Rhizobium loti, NZP2213. Arch Microbiol.; 163: 345-51.

Sonesson A, Jantzen E, Bryn K, Tangen T, Eng J, Zähringer U. (1994) Composition of 23-dihydroxy fatty acid-containig lipopolysaccharides from Legionella israelensis Legionella maceachernii and 
Legionella micdadei. Microbiology.; 140: 1261-71.

Tighe SW, de Lajudie P, Dipietro K, Lindström K, Nick G, Jarvis BDW. (2000) Analysis of cellular fatty acids and phenotypic relationship of Agrobacterium, Bradyrhizobium, Mesorhizobium, Rhizobium and Sinorhizobium species using the Sherlock Microbial Identification System. Int J Syst Bacteriol.; 50: 787-801.

Tsai CM, Frasch CE. (1982) A sensitive silver-staining for detecting lipopolysaccharides in polyacrylamide gels. Anal Biochem.; 119: $115-9$.

Urbanik-Sypniewska T, Choma A, Kutkowska J, Kamińska T, Kandefer-Szerszeń M, Russa R, Dolecka J. (2000) Cytokine inducing activities of rhizobial and mesorhzobial lipopolysaccharides of different lethal toxity. Immunol Exp Clin.; 202: 408-20.
Vincent M. (1970) A manual for the practical study of root-nodule bacteria. International Biological Programme Handbook no15 Blackwell, Oxford, Edinburgh.

Wang ET, van Berkum P, Sui XH, Beyene Chen WX, Martinez-Romero E. (1999) Diversity of rhizobia associated with Amorpha fruticosa isolated from Chinese soils and description of Mesorhizobium amorphae sp. nov. Int $J$ Syst Bacteriol.; 49: 51-65.

Wollenweber HW, Rietschel ET. (1990) Analysis of lipopolysaccharide (lipid A) fatty acids. $J$ Microbiol Methods.; 11: 195-211.

Yokota A, Sakane T, Ophel K, Sawada H. (1993) Further studies on the cellular fatty acid composition of Rhizobium and Agrobacterium species. Inst Ferm Osaka Res Commun.; 16: 86-94. 